\section{Virtual medical plant modeling based on L-system}

Dehong Ding ${ }^{1,2}$, Kui Fang ${ }^{1}$, Song Jing ${ }^{1}$, Liu Bo ${ }^{1}$, Qiao Bo ${ }^{1}$, Hexing $\mathrm{Yu}^{3}$

1. College of Information Science and Technology, Hunan Agricultural University, Changsha 410128, China. 2. HeZhou University, College of Computer Science \& Information Technology, Hezhou, China, 542800 3. College of Resource and Environment, Hunan Agricultural University, Changsha 410128, China

\section{Abstract}

Background: Searching the drug molecules from the medicinal plants become more and more popular given that herbal components have been widely considered to be safe.

In medical virtual plant studies, development rules are difficult to be extracted, the construction of plant organs is highly dependent on equipment and the process is complicated.

Aim: To establish three-dimensional structural virtual plant growth model.

Aim: To establish three-dimensional structural virtual plant growth model.
Methods: The quasi-binary tree structure and its properties were obtained through the research of theory on binary tree, Methods: The quasi-binary tree structure and its properties were obtained through the research of theory on binary tree,
then the relationship between quasi-binary tree structure and plant three-dimensional branching structure model was anathen the relationship between quasi-binary tree structure and plant three-
lyzed, and the three-dimensional morphology of plants was described.

Results: A three-dimensional plant branch structure pattern extracting algorithm based on quasi-binary tree structure. By using 3-D L-system method, the extracted rules were systematized, and standardized. Further more, we built a comprehensive L-model system. With the aid of graphics and PlantVR, we implemented the plant shape and 3-D structure's reconstruction.

Conclusion: Three-dimensional structure virtual plant growth model based on time- controlled L-system has been successfully established.

Keywords: Drug R\&D, toxicity, medical plants, fractals; L-system; quasi binary-trees.

DOI: http://dx.doi.org/10.4314/ahs.v14i4.37

\section{Introduction}

For the sciences of drug R\&D processes, the incentives to undertake $\mathrm{R} \& \mathrm{D}$ activity increase, but the productivity of drug R\&D declines ${ }^{1,2}$. Recently, the attrition rates have sharply risen, especially in late-phase clinical trials $^{3,4}$. The toxicity and other adverse effects of new chemical entities (NCEs) remain to be the major causes to limit the R\&D of new drugs ${ }^{5}$. High safety of herbs make that the R\&D of herbal components become more and more popular.

Understanding of the development of medicinal plants is very important for know what potential drug molecules were contained in medicinal plants. Plant devel-

\section{Corresponding author:}

Kui Fang,

College of Information Science

and Technology, Hunan Agricultural University, Changsha 410128, China.

E-mail:pou9874@126.com
Modulus for Annual Crop Simulation (ACROS). These models, however, are primarily theoretical.
In this study, based on researches of binary tree, structure and properties of quasi-binary tree are addressed. The paper thoroughly analyzed the relation between quasi-binary tree structure and three-dimensional plant branch structure models, and briefly described the threedimensional morphology of plants. On this basis, an algorithm to extract the patterns of three-dimensional plant branch structure based on quasi-binary tree is proposed for the reconstruction of three-dimensional plant morphology. At last, a three-dimensional structural virtual plant growth model based on time-controlled L-system is established.

\section{Methods and Materials}

Three-dimensional Virtual Plant Reconstruction Based On Quasi-binary Tree

To use the L-system, variation patterns and growth features of plants and their organs need to be extracted. Since L-system can capture the topology of plants and convert it into computer discernible languages, specific symbols can be used to denote the organs of plants, axiom $(\omega)$ to describe initial state of plants, and productions $(\mathrm{P})$ to describe the variation patterns of plants and organs, thereby establishing correlated development rules. However, some defects exist in L-system and make its describing process non-intuitive and contain many uncertain factors, for example: L-system uses productions (i.e.: development rules) to establish correlation model, and its description of development rules generally base on growth processes. For relatively complex branching plants or plants of certain species, L-system is inefficient in, sometimes incapable of, describing their development rules.

In order to construct effective three-dimensiona branch structure of plants, on the basis of systematic and thorough study on binary tree-related theories, a method of extracting development rules of virtual branching plants based on quasi-binary tree structure is proposed in this study. It is also a three- dimensional virtual plant morphology reconstruction method. In this section when it come into realization, basic morphology and branching patterns of plants are first analyzed from the botany point of view, growing rules of plants are addressed using quasi-binary tree theory, combining three- dimensional L-system, productions (grammar) of the development rules are constructed, simulative realization of the method is then performed.

Three-dimensional plant branch structures of quasi-binary tree structure uasi-binary tree structure is an extension of binary Qree structure. It is composed of binary tree, ternary tree, and other multi-branch tree structures. Ternary and multi-branch tree structures can be described with pertinent properties of binary tree structure. In a complicated structure with multiple types of branching, these branching structures can be described and organized separately in order to obtain properties of the whole structure.

Definition 1 Quasi-binary trees have the structure of binary tree, with also a parent node and child nodes, and one parent node can generate more than one child node. Binary tree is composed of one parent node and two child nodes. Thus, quasi-binary tree refers to trees with binary tree structure composed of multiple types of branching. Binary tree is a special case of quasi-binary tree. Furthermore, based on the properties of binary tree, properties of other multi-branch trees, for example ternary tree or quaternary tree, can be deduced. Take binary tree as an example, quasi-binary has the following properties:

In a quasi-binary tree with the depth of $\mathrm{k}$, the formula below calculates its number of nodes.

$\mathrm{Sn}=\sum($ muni $\times \mathrm{i}) \mathrm{i}=1 \leq 1 \leq \mathrm{n} \leq \mathrm{var}$

It is the product of the number of different branching types and the number of branches, where, muni indicates the number of different branches. This indicates that properties of a quasi-binary tree are closely related to the number of branch types it contains, i.e. the properties of quasi-binary tree are an integration of other branch-structure trees.

\section{Results and discussion}

Relation between quasi-binary tree structure and hree-dimensional plant branch structure model

It can be seen from Figure. 1 and .2 that three-dimensional model of branching plants is actually a quasibinary tree structure in 3-D space. A logical one-to-one relationship exist between the nodes of the three-dimensional model and the nodes of the quasi-binary tree, which means that three- dimensional model of branching plants have pertinent properties of quasibinary tree structure. In data structure, binary tree is a two-dimensional structure; when it is used to construct three-dimensional models, only revolve and other operations are needed in 3-D space, as shown in Figure.1. 
Figure.1 Three-dimensional plant model.

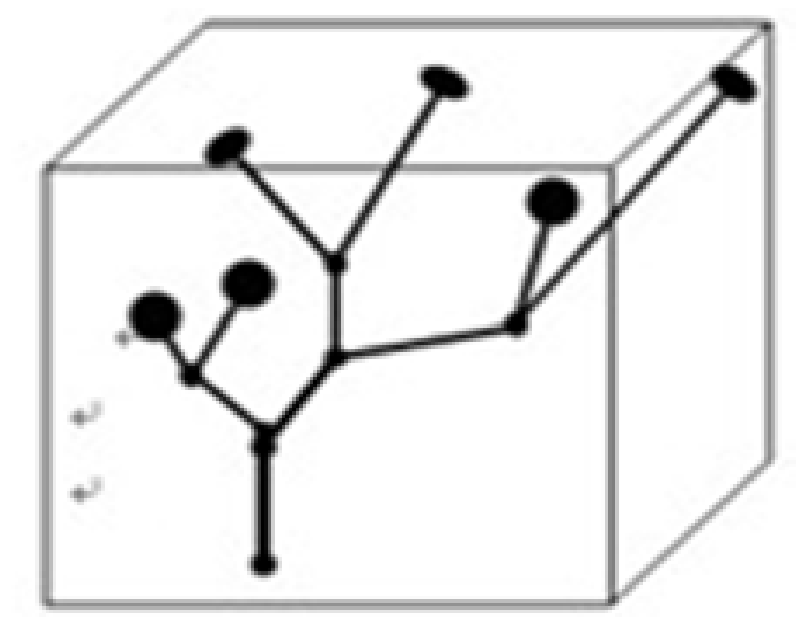

Therefore, the basic idea of constructing fractal- and binary tree-based three-dimensional plant growth model is to always keep two branches during iteration.

Three-dimensional plant branch structure pattern extracting method based on quasi-binary tree.

in good illumination conditions using digital camera or other equipment, save in bmp, raw or jpg formats.

Step 2: Extract the general structures of collected prototypical trees and construct corresponding quasi-binary tree structure models, i.e. construct general logical structures of branching plants;

Step 3: After establishing the quasi-binary tree strucBranching plants generally exhibit branch structures of ture models, address branching types, adjust the model binary branching, ternary branching, and multi-branch- and organize each type of branching structures. In this ing. In order to effectively extrant branching topology of paper, B denotes binary branching and $\mathrm{T}$ denotes plants, the following detailed extracting process is based ternary branching;

on the relation between quasi-binary tree structure and Step 4: Simplify the quasi-binary tree structure models three-dimensional plant branch structure model. until simplest branches as shown in Figure.2 are obStep 1: Collect prototypical photos of branching trees tained;

\section{Figure.2 Quasi-binary tree with 3D structure}

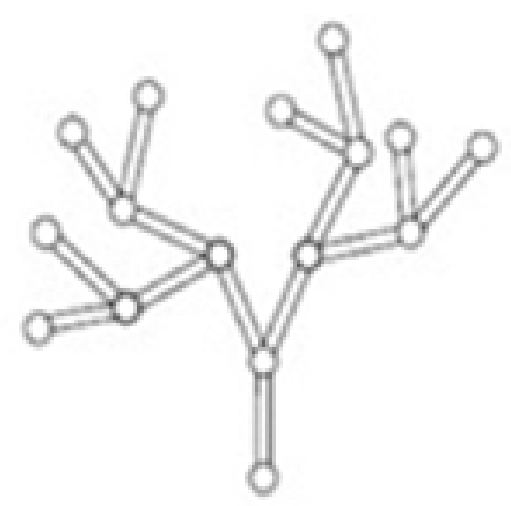

Step 5: Identify the basic iteration rules of each type of Step 7: Improve the basic iteration rules based on the branching ( $\mathrm{B}$ and $\mathrm{T}$, etc.) and their axioms $\mathrm{w}$; key factors and establish complete productions;

Step 6: Based on binary tree structure and the proto- Step 8: Establish the complete three-dimensional Ltypical branching trees, identify key factors including system model; The iteration process is presented in Figbranch length $F(\mathrm{~s})$, initial radius !(w), revolve angle ure.3. $(\theta)$;

\section{Figure.3 Simplification of quasi-binary tree structures and the iteration rules}

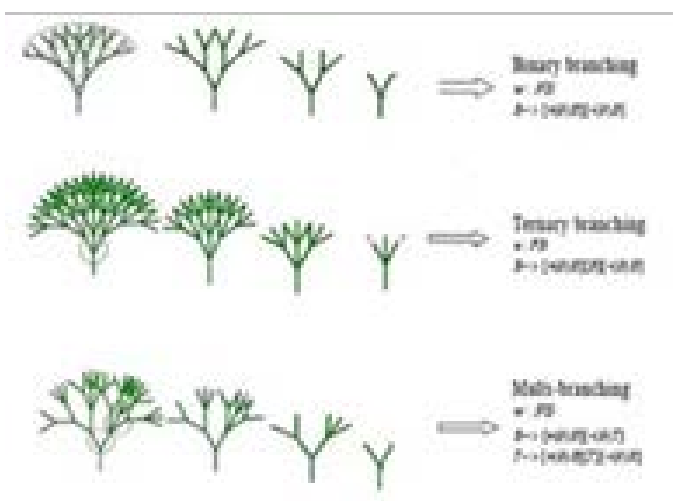

With the method introduced above, related indicators, including branching type var and number of layers $n$ can be obtained. According to the properties of quasibinary trees, the number of nodes is:

$$
\left.\mathrm{Sn}=\sum_{\mathrm{i}=1}^{\mathrm{n}(\text { muni }} \times \mathrm{i}\right)
$$

Where, $1 \leq \mathrm{n} \leq$ var, and muni indicates the number of different branches, it is the product of the number of different branching types and the number of branches.

Therefore, L-system could be defined as:

1) Number of iteration $n$ : the number of layers of the quasi-binary tree structure; (2) Axiom $\mathrm{w}$ : the simplest branches;

(3) Describing process of development rules (productions) $\mathrm{p}:$ First, identify basic development rules of each type of branches based on branching type var ; for example, for binary tree, development rule is $[+(\partial)$ $\mathrm{B}][-(\partial) \mathrm{B}]$, while for ternary trees, $[+(\partial) \mathrm{T}][\mathrm{T}][-(\partial)$ $\mathrm{T}]$, where $\partial$ indicates the revolve angle in 3-D space. Then, represent the three-dimensional spatial location of nodes in each layer with the three vectors $[\mathrm{H}, \mathrm{L}, \mathrm{U}]$ of three-dimensional L-system, and construct development rules (productions) that are reasonable and match the prototypical trees.

The process of describing development rules (produc- tions) demonstrates that the number and structure of development rules (productions) $\mathrm{p}$ are decided by different types of branching trees and the three-dimensional spatial locations of the nodes in each layer. The number of development rules

can be expressed as:

$$
\operatorname{var} \leq \mathrm{p}(\mathrm{i})<2
$$

$\mathrm{n}-1$

$$
-1 \quad\left(\mathrm{i} \square \mathrm{R}^{*}\right)(1)
$$

Apparently, more types of branches and greater number of branches means more development rules (productions). The larger the number of growth units, the more complex the generated three- dimensional structure, vice versa.

In L-system, the describing of development rules (productions) is closely related to plant growth processes8, and the number of development rules and structural descriptions are uncertain. This can be

expressed as:

$\operatorname{var} \leq p(i)<\infty$

$\left(\mathrm{i} \square \mathrm{R}^{*}\right)(2)$

Comparing the describing process of development rules (productions) p with Equation (1) and (3), it can 
be seen that no matter for the number of development Original image, we found that, this method can describe rules (productions) or the describing of each rule, the proposed method has good operability and certainty.

Simulation of Three-Dimensional Plant Branch Structures of Quasi-binary Tree Structure

To prove the effectiveness of extraction algorithm on

Tranch plants 3-D topology with quai- binary tee fen- The C++ definition as below: Typedef struct TeeNoture presented above, we selected two sets of data to de\{ unsigned Index; int Lay; // Number of iteration compare in the contest: take photos for two distinct char Axiom; // axiom char rule[num]; // Producbranching pattern respectively (dichotomous branching tions (num means numbers of branches) char $\operatorname{Var}[\mathrm{n}]$ and Mixed branching), then extracted plant branching // Branching species (Including dichotomous branchgrowth rules and transform it into three-dimensional ing, trigeminal branching and multiple Branching) float L-System rules(productions) with the method men- Angle[n]; // The angle of rotation in three-dimensiontioned above in the study, develop simulation system al space char param[MAXPARAM][256];;; Figure .4(a) of reconstruction about three-dimensional branching and .5(a) are images of two plants (samples) that have structure of plant, in MFC IDE, and take simulation binary branching structure and multi-branching strucdraw in the system. Contrast the simulation image and ture, respectively.

Figure.4 Plant with binary branching structure and its simulation result. (A) Plant with binary branching structure; (B) Simulation result

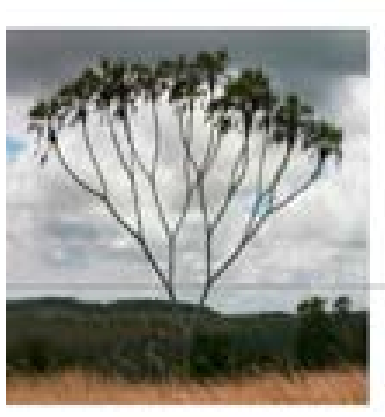

(A)

Fig. 4

For these two samples, the related data as shown in table 1 the proposed method is applied to extract development rules of the branches and convert to three- dimensional L-system rules, and then perform three-dimensional reconstruction, and the results are presented in Figure .4(b) and .5(b). $\begin{array}{ll}\text { Comparison between reconstructed three-dimensional } & \text { opment rules (productions) of the plants, and effective- } \\ \text { images and original images indicates that the proposed } & \text { ly describe development rules of plants with specific }\end{array}$ images and original images indicates that the proposed ly describe development rules of plants with specific method could rapidly and accurately extract the devel- $\begin{aligned} & \text { types of branch } \\ & \text { construction. }\end{aligned}$

Figure.5 Plant with multi-branching structure and its simulation result. (a) Plant with multi-branching structure(b) Simulation result

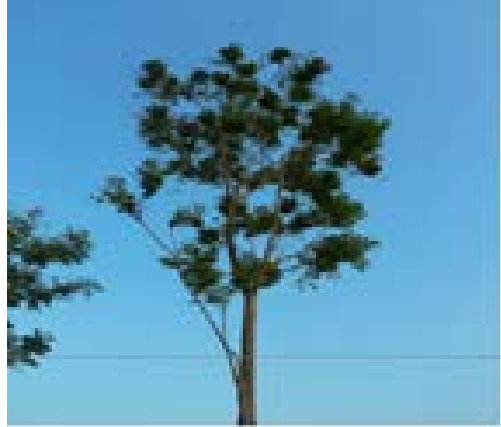

(A)

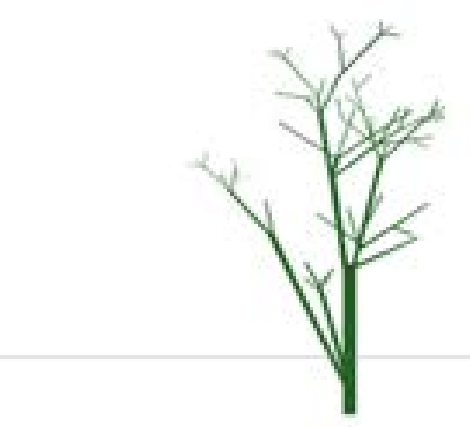

(B)

Fig. 5

\section{Conclusions}

The present study provides a new method based on quasi-binary tree structure of a virtual three-dimensional reconstruction of plant branching, which overcome the existed defective of methods and theories. The simulation results indicate that, this method overcomes the non-intuitive and uncertainty about description of plant growth rules and definitions effectively, elaborated branching plant growth rule description operability, provides a new way to describe the plant growth regulation. However, because of the complexity of plant growth structure, this method needs artificial recognition when building quasi-binary tree models, which influences the applicability, the next research focuses on automatic improvement about construction of quasi-binary tree model, make it able to distinguish the branching of plant and establish correct quasi-binary tree structure automatically.

\section{Acknowledgement}

This work was supported by General scientific research projects in Hunan Province department of education, China (Grant No.14C0542), Graduate student research innovation project of Hunan Province (Grant No CX2014B306) and Guangxi Experiment Centre of Science and Technology (Grant No:LGZXKF201112). 


\section{References}

1. Lanjouw, J. \& M. Schankerman (2004) Econ. J. 114: 44165.

2. Jones, B. (2009) Rev. Econ. Stat. 76: 283-317.Q

3. Mervis, J. (2005) Science 309: 726.

4. David, E., T. Tramontin, \& R. Zemmel (2009) Nature Rev. Drug Discov. 8: 609-10.
5. Li, A.P. (2014) Curr. Top Med. Chem. 14(11):132538.

6. Ashiq, S., M. Hussain \& B. Ahmad (2014) Fungal. Genet. Biol. 66: 1-10.

7.Williams, J.R., K.G. Renard \& P.T. Dyke. (1983) Journal of Soil and Water Conservation 38(5): 381-3.

8. Zhao, C., G. Wang, \& X. Guo (2007) China. J. Transactions of the CSAE. 23(9):1-6. 